\title{
Erratum
}

\section{Horospheres on Abelian Covers}

\section{François Ledrappier}

Volume 28, N. 2, (1997) pp. 363-375

A preliminary version of the paper $[\mathbf{L}]$ was inadvertently printed. The statements there are true and proven only if the words "proper" and "discrete" are given more restricted acceptations.

Namely, Theorems A and B are correct if proper means proper leaf, i.e. locally closed submanifold, instead of closed submanifold, as claimed page 363. Besides, theorems A' and B', A" and B" and proposition 3' are all correct if discrete means discrete for the induced topology, instead of discrete subset.

The equivalence between the leaf $W^{s s}$ being proper and the $\bar{\Gamma}$-orbit of $W^{s s}$ being discrete (on the top of page 372 ) still holds with these new acceptations, since both properties are equivalent to the subset $\bar{\Gamma} W^{s s}$ being locally closed in $T^{1} \bar{M}$.

I thank Mark Pollicott for pointing out this error to me and for providing a counterexample. I take this opportunity to mention that Proposition 2 can also be directly obtained from [E], Theorem 5.2.

\section{References}

[E] P. Eberlein, Geodesic flow on negatively curved manifolds II, T.A.M.S.. 178 (1973), 57-82.

[L] F. Ledrappier Horospheres on abelian covers, Bull.. Braz. Math. Soc. 28(2) (1997) , 363-375. 